Journal of Bangladesh Academy of Sciences, Vol. 36, No. 2, 187-197, 2012

\title{
APPROXIMATE SOLUTION OF FOURTH ORDER NEAR CRITICALLY DAMPED NONLINEAR SYSTEMS WITH SPECIAL CONDITIONS
}

\author{
MD. ASRAFUL ALOM* AND M. ALHAZ UDDIN \\ Department of Mathematics, Khulna University of Engineering \& Technology, \\ Khulna-9203, Bangladesh
}

\begin{abstract}
A perturbation technique has been developed based on the Krylov-Bogoliubov-Mitropolskii (KBM) method to investigate the solution of fourth order near critically damped nonlinear systems in the case of $\lambda_{1} \rightarrow \lambda_{2}, \quad \lambda_{4} \approx \lambda_{3}+2 \lambda_{1}$ but $\lambda_{4}\left\langle 2 \lambda_{3}\right.$ among the eigenvalues $\lambda_{1}, \lambda_{2}, \lambda_{3}, \lambda_{4}$. The solutions obtained by this technique were compared with those obtained by numerical method. The method has been explained by an example.
\end{abstract}

Key words: Perturbation method, Weak nonlinearity, Near critically damped nonlinear system

\section{INTRODUCTION}

The Krylov-Bogoliubov-Mitropolskii method (Krylov and Bogoliubov 1947, Bogoliubov and Mitropolskii 1961) is one of the widely used techniques to obtain analytical approximate solution of weakly nonlinear systems and this method was originally developed for finding periodic solutions of nonlinear systems with small nonlinearities. The method was extended by Popov (1956) to damped oscillatory systems. Murty et al. (1969) investigated an over-damped nonlinear system using Bogoliubov's method. Murty (1971) presented a unified KBM method for solving second order nonlinear systems which cover the un-damped, damped and over-damped cases. Alam and Sattar (1996) extended the KBM method for third order critically damped nonlinear systems. Alam (2002) also investigated the solution of third order nonlinear systems when two of the eigenvalues are almost equal and the other is small. Haque et al. (2011) investigated the solution of fourth order critically damped oscillatory nonlinear systems when two of the eigenvalues are real and equal and the other two are complex conjugate. Akbar et al. (2007) extended the KBM method for solving fourth order more critically damped nonlinear systems. Recently, Rahman et al. (2009) developed a technique for solving of fourth order near critically damped nonlinear systems. For the relation $\lambda_{4} \approx \lambda_{3}+2 \lambda_{1}$, the solution obtained in Rahman et al. (2009) broke-down. The aim of this article was to fill this gap, that is, the authors were interested to investigate the solution when the relation $\lambda_{4} \approx \lambda_{3}+2 \lambda_{1}$ exists among the eigenvalues $\lambda_{1}, \lambda_{2}, \lambda_{3}, \lambda_{4}$. The solutions obtained by this technique showed good coincidence with those obtained by numerical method.

\footnotetext{
*Corresponding author: <asraf.math.kuet@gmail.com>, <alhazuddin@yahoo.com>.
} 


\section{MATERIALS AND METHOD}

Let us consider the fourth order weakly nonlinear ordinary differential equation in the following form

$$
\frac{d^{4} x}{d t^{4}}+c_{1} \frac{d^{3} x}{d t^{3}}+c_{2} \frac{d^{2} x}{d t^{2}}+c_{3} \frac{d x}{d t}+c_{4} x=-\varepsilon f(x),
$$

where $\varepsilon$ is a positive small parameter, $c_{1}, c_{2}, c_{3}, c_{4}$ are constants and $f(x)$ is the given nonlinear function. The constants are defined in terms of the eigenvalues $-\lambda_{i}$, $i=1,2,3,4$ of the unperturbed Eq. of Eq. (1) as

$$
c_{1}=\sum_{i=1}^{4} \lambda_{i}, \quad c_{2}=\sum_{\substack{i, j=1 \\ i \neq j}}^{4} \lambda_{i} \lambda_{j}, \quad c_{3}=\sum_{\substack{i, j, k=1 \\ i \neq j \neq k}}^{4} \lambda_{i} \lambda_{j} \lambda_{k}
$$

and $c_{4}=\prod_{i=1}^{4} \lambda_{i}$.

The Eq. (1) becomes linear when $\varepsilon=0$, and suppose the eigenvalues $-\lambda_{1}$ and $-\lambda_{2}$ are almost equal $\left(\lambda_{1} \approx \lambda_{2}\right)$ and other two eigenvalues $-\lambda_{3}$ and $-\lambda_{4}$ are distinct. Therefore, the unperturbed solution is

$$
x(t, 0)=\frac{1}{2} a_{1,0}\left(e^{-\lambda_{1} t}+e^{-\lambda_{2} t}\right)+a_{2,0}\left(\frac{e^{-\lambda_{1} t}-e^{-\lambda_{2} t}}{\lambda_{1}-\lambda_{2}}\right)+a_{3,0} e^{-\lambda_{3} t}+a_{4,0} e^{-\lambda_{4} t},
$$

where $a_{i, 0}(i=1,2,3,4)$ are arbitrary constants.

When $\varepsilon \neq 0$, following Alam (2002) technique we choose the solution of Eq. (1) in the form

$$
\begin{aligned}
x(t, \varepsilon)= & \frac{1}{2} a_{1}(t)\left(e^{-\lambda_{1} t}+e^{-\lambda_{2} t}\right)+a_{2}(t)\left(\frac{e^{-\lambda_{1} t}-e^{-\lambda_{2} t}}{\lambda_{1}-\lambda_{2}}\right)+a_{3}(t) e^{-\lambda_{3} t} \\
& +a_{4}(t) e^{-\lambda_{4} t}+\varepsilon u_{1}\left(a_{1}, a_{2}, a_{3}, a_{4}, t\right)+\varepsilon^{2} \ldots,
\end{aligned}
$$

where $a_{i}(i=1,2,3,4)$ satisfy the following first order differential equations:

$$
\frac{d a_{i}(t)}{d t}=\varepsilon A_{1}\left(a_{1}, a_{2}, a_{3}, a_{4}, t\right)+\varepsilon^{2} \cdots, \quad i=1,2,3,4
$$

Confining only to a first few terms $1,2,3, \ldots, n$ in the series expansions Eqs. (3) and (4), we calculate the functions $u_{1}$ and $A_{i}, i=1,2,3,4$ such that $a_{i}(t), i=1,2,3,4$ appearing in Eqs. (3) and (4) satisfy the given differential Eq. (1) with an accuracy of $\operatorname{order} \varepsilon^{n+1}$. To determine the unknown functions $u_{1}, A_{1}, A_{2}, A_{3}, A_{4}$, it is assumed (as customary in the KBM method) that the correction term $u_{1}$ does not contain secular-type term $t e^{-\lambda_{i} t}$, which make them large. Differentiating Eq. (3) four times with respect to $t$, 
substituting the derivatives $\frac{d^{4} x}{d t^{4}}, \frac{d^{3} x}{d t^{3}}, \frac{d^{2} x}{d t^{2}}, \frac{d x}{d t}$ and $x$ in the original Eq. (1), utilizing the relations presented in Eq. (4) and finally equating the coefficients of $\varepsilon$, we obtain

$$
\begin{aligned}
& \frac{1}{2}\left(e^{-\lambda_{1} t}\left(D-\lambda_{1}+\lambda_{2}\right)\left(D-\lambda_{1}+\lambda_{3}\right)\left(D-\lambda_{1}+\lambda_{4}\right)+e^{-\lambda_{2} t}\left(D-\lambda_{2}+\lambda_{1}\right)\left(D-\lambda_{2}+\lambda_{3}\right)\left(D-\lambda_{2}+\lambda_{4}\right)\right) A_{1} \\
& +\left(D+\lambda_{4}\right)\left(e^{-\lambda_{1} t}\left(\lambda_{1}-\lambda_{3}-\frac{3}{2} D\right)+e^{-\lambda_{2} t}\left(\lambda_{2}-\lambda_{3}-\frac{3}{2} D\right)\right) A_{2}+e^{-\lambda_{3} t}\left(D-\lambda_{3}+\lambda_{1}\right)\left(D-\lambda_{3}+\lambda_{2}\right) \times \\
& \left(D-\lambda_{3}+\lambda_{4}\right) A_{3}+e^{-\lambda_{4} t}\left(D-\lambda_{4}+\lambda_{1}\right)\left(D-\lambda_{4}+\lambda_{2}\right)\left(D-\lambda_{4}+\lambda_{3}\right) A_{4}+\left(\frac{e^{-\lambda_{1} t}-e^{-\lambda_{2} t}}{\lambda_{1}-\lambda_{2}}\right)\left(D+\lambda_{4}\right) D \times \\
& \left(D+\lambda_{3}-\frac{\lambda_{1}+\lambda}{2}\right) A_{2}-\left(\frac{\lambda_{1} e^{-\lambda_{1} t}-\lambda_{2} e^{-\lambda_{2} t}}{\lambda_{1}-\lambda_{2}}\right) D\left(D+\lambda_{3}-\frac{\lambda_{1}+\lambda}{2}\right) A_{2}+\left(D+\lambda_{1}\right)\left(D+\lambda_{2}\right)\left(D+\lambda_{3}\right) \times \\
& \left(D+\lambda_{4}\right) u_{1}=-f^{(0)},
\end{aligned}
$$

where $f^{(0)}=f\left(x_{0}\right)$ and $x_{0}=\frac{1}{2} a_{1}(t)\left(e^{-\lambda_{1} t}+e^{-\lambda_{2} t}\right)+a_{2}(t)\left(\frac{e^{-\lambda_{1} t}-e^{-\lambda_{2} t}}{\lambda_{1}-\lambda_{2}}\right)+a_{3}(t) e^{-\lambda_{3} t}+a_{4}(t) e^{-\lambda_{4} t}$.

It is assumed that the function $f^{(0)}$ can be expanded in power series (Taylor's series) in the form (Bogoliubov and Mitropolskii 1961 for details)

$$
f^{(0)}=\sum_{r=0}^{n} F_{r}\left(a_{3} e^{-\lambda_{3} t}, a_{4} e^{-\lambda_{4} t}\right)\left\{\frac{1}{2} a_{1}\left(e^{-\lambda_{1} t}+e^{-\lambda_{2} t}\right)+a_{2}\left(\frac{e^{-\lambda_{1} t}-e^{-\lambda_{2} t}}{\lambda_{1}-\lambda_{2}}\right)\right\}^{r}
$$

where $n$ is the order of polynomial of the nonlinear function $f$. This assumption is certainly valid when $f$ is a polynomial function of $x$. Such polynomial functions cover some special and important systems in mechanics. Following Alam's (2002), in this paper the authors assumed that $u_{1}$ does not contain the terms $F_{0}$ and $F_{1}$ of $f^{(0)}$, since the system is considered to near critically damped. Substituting the value of $f^{(0)}$ from Eq. (6) into Eq. (5) and equating the coefficients of like powers of $\left(\frac{e^{\lambda_{1} t}-e^{\lambda_{2} t}}{\lambda_{1}-\lambda_{2}}\right)$, the present authors obtained

$$
\begin{aligned}
& e^{-\lambda_{3} t}\left(D-\lambda_{3}+\lambda_{1}\right)\left(D-\lambda_{3}+\lambda_{2}\right)\left(D-\lambda_{3}+\lambda_{4}\right) A_{3}+e^{-\lambda_{4} t}\left(D-\lambda_{4}+\lambda_{1}\right)\left(D-\lambda_{4}+\lambda_{2}\right)\left(D-\lambda_{4}+\lambda_{3}\right) A_{4} \\
& +\frac{1}{2}\left\{e^{-\lambda_{1} t}\left(D-\lambda_{1}+\lambda_{2}\right)\left(D-\lambda_{1}+\lambda_{3}\right)\left(D-\lambda_{1}+\lambda_{4}\right)+e^{-\lambda_{2} t}\left(D-\lambda_{2}+\lambda_{1}\right)\left(D-\lambda_{2}+\lambda_{3}\right) \times\right. \\
& \left.\left(D-\lambda_{2}+\lambda_{4}\right)\right\} A_{1}+\left(D+\lambda_{4}\right)\left\{e^{-\lambda_{1} t}\left(\lambda_{1}-\lambda_{3}-\frac{3}{2} D\right)+e^{-\lambda_{2} t}\left(\lambda_{2}-\lambda_{3}-\frac{3}{2} D\right)\right\} A_{2}-\left(\frac{\lambda_{1} e^{-\lambda_{1} t}-\lambda_{2} e^{-\lambda_{2} t}}{\lambda_{1}-\lambda_{2}}\right) \\
& \times D\left(D+\lambda_{3}-\frac{\lambda_{1}+\lambda_{2}}{2}\right) A_{2}=-F_{0}-\frac{1}{2} a_{1}\left(e^{-\lambda_{1} t}+e^{-\lambda_{2} t}\right) F_{1}
\end{aligned}
$$




$$
D\left(D+\lambda_{4}\right)\left(D+\lambda_{3}-\frac{\lambda_{1}+\lambda_{2}}{2}\right) A_{2}=-a_{2} F_{1},
$$

and

$$
\begin{aligned}
& \left(D+\lambda_{1}\right)\left(D+\lambda_{2}\right)\left(D+\lambda_{3}\right)\left(D+\lambda_{4}\right) u_{1} \\
& =-\sum_{r=2}^{n} F_{r}\left(a_{3} e^{-\lambda_{3} t}, a_{4} e^{-\lambda_{4} t}\right) \times\left\{\frac{1}{2} a_{1}\left(e^{-\lambda_{1} t}+e^{-\lambda_{2} t}\right)+a_{2}\left(\frac{e^{-\lambda_{1} t}-e^{-\lambda_{2} t}}{\lambda_{1}-\lambda_{2}}\right)\right\}^{r} .
\end{aligned}
$$

KBM (Krylov and Bogoliubov 1947, Bogoliubov and Mitropolskii 1961), Alam and Sattar (1996) and Alam (2002) have imposed the condition that $u_{1}$ does not contain the fundamental terms (the solution presented in Eq. (2) is called generating solution and its terms are called fundamental terms) of $f^{(0)}$. The solution of Eq. (8) gives value of the unknown function $A_{2}$. If the nonlinear function $f$ and the eigenvalues $-\lambda_{1},-\lambda_{2},-\lambda_{3},-\lambda_{4}$ of the corresponding linear equation of Eq. (1) are not specified then it is not easy to solve the Eq. (7) for the unknown functions $A_{1}, A_{3}$ and $A_{4}$. When these are specified, the values of $A_{1}, A_{3}$ and $A_{4}$ can be found subject to the condition that the coefficients in the solutions of $A_{1}, A_{3}$ and $A_{4}$ do not become large (Akbar et al. 2007, Alam and Sttar 1996, Alam 2002 for details), as if $A_{1}, A_{3}$ and $A_{4}$ do not contain terms involving $t e^{-t}$. In this article, we have imposed the conditions that the relation $\lambda_{4} \approx \lambda_{3}+2 \lambda_{1}$ but $\lambda_{4}\left\langle 2 \lambda_{3}\right.$ exists among the eigenvalues $\lambda_{1}, \lambda_{3}, \lambda_{4}$ (also $\lambda_{1} \rightarrow \lambda_{2}$ since the system is near critically damped). These relations are important, because under these relations the coefficients in the solutions of $A_{1}, A_{3}$ and $A_{4}$ do not become large. Under these imposed conditions, the authors obtained the values of $A_{1}, A_{3}$ and $A_{4}$ from Eq. (7). Substituting the values of $A_{1}, A_{2}, A_{3}$ and $A_{4}$ in the Eq. (4), the authors obtained the solutions of $\frac{d a_{i}}{d t}(i=1,2,3,4)$, which are proportional to the small parameter $\varepsilon$.

So these are slowly varying functions of time $t$, that is, these are almost constants and by integrating the values of $a_{i}(i=1,2,3,4)$ are obtained. It is laborious work to solve the Eq. (9) for $u_{1}$. However, as $\lambda_{1} \rightarrow \lambda_{2}$ it takes the following simple form

$$
\left(D+\lambda_{1}\right)^{2}\left(D+\lambda_{3}\right)\left(D+\lambda_{4}\right) u_{1}=-\sum_{r=2}^{n} F_{r}\left(a_{3} e^{-\lambda_{3} t}, a_{4} e^{-\lambda_{4} t}\right)\left\{e^{-\lambda_{1} t}\left(a_{1}-a_{2} t\right)\right\}^{r} .
$$

Solving Eq. (10), we obtain the value of $u_{1}$. Finally, substituting the values of $a_{i}(i=1,2,3,4)$ and $u_{1}$ in the Eq. (3), we obtain the complete solution of Eq. (1).

\section{EXAMPLE}

For an example of the above method, we consider the following fourth order nonlinear differential equation, 
$\frac{d^{4} x}{d t^{4}}+c_{1} \frac{d^{3} x}{d t^{3}}+c_{2} \frac{d^{2} x}{d t^{2}}+c_{3} \frac{d x}{d t}+c_{4} x=-\varepsilon x^{3}$.

Here $f(x)=x^{3}$ and $x_{0}=\frac{1}{2} a_{1}\left(e^{-\lambda_{1} t}+e^{-\lambda_{2} t}\right)+a_{2}\left(\frac{e^{-\lambda_{1} t}-e^{-\lambda_{2} t}}{\lambda_{1}-\lambda_{2}}\right)+a_{3} e^{-\lambda_{3} t}+a_{4} e^{-\lambda_{4} t}$.

Thus, $f^{(0)}=\left\{\frac{1}{2} a_{1}\left(e^{-\lambda_{1} t}+e^{-\lambda_{2} t}\right)+a_{2}\left(\frac{e^{-\lambda_{1} t}-e^{-\lambda_{2} t}}{\lambda_{1}-\lambda_{2}}\right)+a_{3} e^{-\lambda_{3} t}+a_{4} e^{-\lambda_{4} t}\right\}^{3}$,

$$
F_{0}=\left(a_{3} e^{-\lambda_{3} t}+a_{4} e^{-\lambda_{4} t}\right)^{3}, F_{1}=3\left(a_{3} e^{-\lambda_{3} t}+a_{4} e^{-\lambda_{4} t}\right)^{2} .
$$

According to the Eqs. (7) - (9), we obtain

$$
\begin{aligned}
& e^{-\lambda_{3} t}\left(D-\lambda_{3}+\lambda_{1}\right)\left(D-\lambda_{3}+\lambda_{2}\right)\left(D-\lambda_{3}+\lambda_{4}\right) A_{3}+e^{-\lambda_{4} t}\left(D-\lambda_{4}+\lambda_{1}\right)\left(D-\lambda_{4}+\lambda_{2}\right) \times \\
& \left(D-\lambda_{4}+\lambda_{3}\right) A_{4}+\frac{1}{2}\left\{e^{-\lambda_{1} t}\left(D-\lambda_{1}+\lambda_{2}\right)\left(D-\lambda_{1}+\lambda_{3}\right)\left(D-\lambda_{1}+\lambda_{4}\right)\right. \\
& \left.+e^{-\lambda_{2} t}\left(D-\lambda_{2}+\lambda_{1}\right)\left(D-\lambda_{2}+\lambda_{3}\right)\left(D-\lambda_{2}+\lambda_{3}\right)\right\} A_{1}+\left(D+\lambda_{4}\right)\left\{e^{-\lambda_{1} t}\left(\lambda_{1}-\lambda_{3}-\frac{3}{2} D\right)\right. \\
& \left.+e^{-\lambda_{2} t}\left(\lambda_{2}-\lambda_{3}-\frac{3}{2} D\right)\right\} A_{2}-\left(\frac{\left(\lambda_{1} e^{-\lambda_{1} t}-\lambda_{2} e^{-\lambda_{2} t}\right)}{\left(\lambda_{1}-\lambda_{2}\right)}\right) D\left(D+\lambda_{3}-\frac{\lambda_{1}+\lambda_{2}}{2}\right) A_{2} \\
& =-\left[\left(a_{3} e^{-\lambda_{3} t}+a_{4} e^{-\lambda_{4} t}\right)^{3}+3\left(a_{3} e^{-\lambda_{3} t}+a_{4} e^{-\lambda_{4} t}\right)^{2} \frac{1}{2} a_{1}\left(e^{-\lambda_{1} t}+e^{-\lambda_{2} t}\right)\right], \\
& D\left(D+\lambda_{4}\right)\left(D+\lambda_{3}-\frac{\lambda_{1}+\lambda_{2}}{2}\right) A_{2}=-3 a_{2}\left(a_{3} e^{-\lambda_{3} t}+a_{4} e^{-\lambda_{4} t}\right)^{2},
\end{aligned}
$$

and

$$
\begin{aligned}
& \left(D+\lambda_{1}\right)\left(D+\lambda_{2}\right)\left(D+\lambda_{3}\right)\left(D+\lambda_{4}\right) u_{1} \\
& =-\sum_{r=2}^{3} F_{r}\left(a_{3} e^{-\lambda_{3} t}, a_{4} e^{-\lambda_{4} t}\right)\left\{\frac{1}{2} a_{1}\left(e^{-\lambda_{1} t}+e^{-\lambda_{2} t}\right)+a_{2}\left(\frac{e^{-\lambda_{1} t}-e^{-\lambda_{2} t}}{\lambda_{1}-\lambda_{2}}\right)\right\}^{r} .
\end{aligned}
$$

Solving Eq. (14), we obtain

$$
A_{2}=a_{2}\left[n_{1} a_{3}^{2} e^{-2 \lambda_{3} t}+n_{2} a_{3} a_{4} e^{-\left(\lambda_{3}+\lambda_{4}\right) t}+n_{3} a_{4}^{2} e^{-2 \lambda_{4} t}\right],
$$

where

$$
\begin{aligned}
& n_{1}=\frac{3}{\lambda_{3}\left(\lambda_{1}+\lambda_{2}+2 \lambda_{3}\right)\left(2 \lambda_{3}-\lambda_{4}\right)}, \quad n_{2}=\frac{12}{\lambda_{3}\left(\lambda_{3}+\lambda_{4}\right)\left(\lambda_{1}+\lambda_{2}+2 \lambda_{4}\right)}, \\
& n_{3}=\frac{3}{\lambda_{4}^{2}\left(\lambda_{1}+\lambda_{2}-2 \lambda_{3}+4 \lambda_{4}\right)} .
\end{aligned}
$$

Substituting the value of $A_{2}$ from Eq. (16) into Eq. (13). In order to separate the Eq. (13) for determining the unknown functions $A_{1}, A_{3}$ and $A_{4}$, we use the conditions as 
discussed in the method (see also Akbar et al. 2007 and Alam 2002). It is to note that our solution approaches toward critically damped solution (see Alam 2002) if $\lambda_{1} \rightarrow \lambda_{2}$. However, Eq. (13) has not an exact solution unless $\lambda_{1} \rightarrow \lambda_{2}$. Now we consider $\lambda_{4} \approx \lambda_{3}+2 \lambda_{1}$ but $\lambda_{4}\left\langle 2 \lambda_{3}\right.$. Under these imposed conditions and by equating like terms on both sides of the Eq. (13), we obtain

$$
\begin{aligned}
& e^{-\lambda_{1} t}\left(D-\lambda_{1}+\lambda_{2}\right)\left(D-\lambda_{1}+\lambda_{3}\right)\left(D-\lambda_{1}+\lambda_{4}\right) A_{1} \\
& =-a_{2} a_{3}^{2} n_{1} \lambda_{2} \lambda_{3}\left(\lambda_{1}+\lambda_{2}+2 \lambda_{3}\right) t e^{-\left(\lambda_{1}+2 \lambda_{3}\right) t}-\frac{1}{2} a_{2} a_{3} a_{4} n_{2} \lambda_{2}\left(2 \lambda_{4}^{2}+2 \lambda_{3} \lambda_{4}+\lambda_{1} \lambda_{3}+\lambda_{2} \lambda_{3}\right. \\
& \left.\quad+\lambda_{1} \lambda_{4}+\lambda_{2} \lambda_{4}\right) t e^{-\left(\lambda_{1}+\lambda_{3}+\lambda_{4}\right) t}-a_{2} a_{4}^{2} n_{3} \lambda_{2} \lambda_{4}\left(\lambda_{1}+\lambda_{2}-2 \lambda_{3}+4 \lambda_{4}\right) t e^{-\left(\lambda_{1}+2 \lambda_{4}\right) t} \\
& e^{-\lambda_{3} t}\left(D-\lambda_{3}+\lambda_{1}\right)\left(D-\lambda_{3}+\lambda_{2}\right)\left(D-\lambda_{3}+\lambda_{4}\right) A_{3} \\
& =\left[a_{2} n_{1}\left\{\left(\lambda_{1}+2 \lambda_{3}\right)\left(\lambda_{1}+2 \lambda_{3}-\lambda_{4}\right)+\lambda_{3}\left(\lambda_{1}+\lambda_{2}+2 \lambda_{3}\right)\right\}-\frac{3}{2} a_{1}\right] a_{3}^{2} e^{-\left(\lambda_{1}+2 \lambda_{3}\right) t} \\
& \quad+\left[a_{2} n_{1}\left(\lambda_{2}+2 \lambda_{3}\right)\left(\lambda_{2}+2 \lambda_{3}-\lambda_{4}\right)-\frac{3}{2} a_{1}\right] a_{3}^{2} e^{-\left(\lambda_{2}+2 \lambda_{3}\right) t}-a_{3}^{3} e^{-3 \lambda_{3} t}-3 a_{3}^{2} a_{4} e^{-\left(2 \lambda_{3}+\lambda_{4}\right) t}
\end{aligned}
$$

and

$$
\begin{aligned}
& e^{-\lambda_{4} t}\left(D-\lambda_{4}+\lambda_{1}\right)\left(D-\lambda_{4}+\lambda_{2}\right)\left(D-\lambda_{4}+\lambda_{3}\right) A_{4} \\
&= {\left[\frac{1}{2} a_{2} n_{2}\left\{\left(\lambda_{1}+\lambda_{3}\right)\left(2 \lambda_{1}+\lambda_{3}+3 \lambda_{4}\right)+\left(2 \lambda_{4}^{2}+2 \lambda_{3} \lambda_{4}+\lambda_{1} \lambda_{3}+\lambda_{2} \lambda_{3}+\lambda_{1} \lambda_{4}+\lambda_{2} \lambda_{4}\right)\right\}\right.} \\
&\left.-3 a_{1}\right] a_{3} a_{4} e^{-\left(\lambda_{1}+\lambda_{3}+\lambda_{4}\right) t}+\left[a_{2} n_{3}\left\{\left(\lambda_{1}+\lambda_{4}\right)\left(\lambda_{1}-\lambda_{3}+3 \lambda_{4}\right)+\lambda_{4}\left(\lambda_{1}+\lambda_{2}-2 \lambda_{3}+4 \lambda_{4}\right)\right\}\right. \\
&\left.-\frac{3}{2} a_{1}\right] a_{4}^{2} e^{-\left(\lambda_{1}+2 \lambda_{4}\right) t}+\left[\frac{1}{2} a_{2} n_{2}\left(\lambda_{2}+\lambda_{3}\right)\left(2 \lambda_{2}+\lambda_{3}+3 \lambda_{4}\right)-3 a_{1}\right] a_{3} a_{4} e^{-\left(\lambda_{2}+\lambda_{3}+\lambda_{4}\right) t} \\
&+\left[a_{2} n_{3}\left(\lambda_{2}+\lambda_{4}\right)\left(\lambda_{2}-\lambda_{3}+3 \lambda_{4}\right)-\frac{3}{2} a_{1}\right] a_{4}^{2} e^{-\left(\lambda_{2}+2 \lambda_{4}\right) t}-\left[3 a_{3} a_{4}^{2} e^{-\left(\lambda_{3}+2 \lambda_{4}\right) t}+a_{4}^{3} e^{-3 \lambda_{4} t}\right] .
\end{aligned}
$$

The particular solutions of Eqs. (18)- (20) yield respectively

$$
\begin{aligned}
A_{1}= & i_{1} a_{2} a_{3}^{2} t e^{-\left(\lambda_{1}-\lambda_{2}+2 \lambda_{3}\right) t}+i_{2} a_{2} a_{3}^{2} t e^{-\left(\lambda_{1}-\lambda_{2}+2 \lambda_{3}\right) t}+i_{3} a_{2} a_{3} a_{4} t e^{-\left(\lambda_{1}-\lambda_{2}+\lambda_{3}+\lambda_{4}\right) t} \\
& +i_{4} a_{2} a_{3} a_{4} t e^{-\left(\lambda_{1}-\lambda_{2}+\lambda_{3}+\lambda_{4}\right) t}+i_{5} a_{2} a_{4}^{2} t e^{-\left(\lambda_{1}-\lambda_{2}+2 \lambda_{4}\right) t}+i_{6} a_{2} a_{4}^{2} t e^{-\left(\lambda_{1}-\lambda_{2}+2 \lambda_{4}\right) t} \\
A_{3}= & \left(M_{1} a_{2}+M_{2} a_{1}\right) a_{3}^{2} e^{-\left(\lambda_{1}+\lambda_{3}\right) t}+\left(M_{3} a_{2}+M_{4} a_{1}\right) a_{3}^{2} e^{-\left(\lambda_{2}+\lambda_{3}\right) t}+M_{5} a_{3}^{3} e^{-2 \lambda_{3} t} \\
& +M_{6} a_{3}^{2} a_{4} e^{-\left(\lambda_{3}+\lambda_{4}\right) t}
\end{aligned}
$$

and

$$
\begin{aligned}
A_{4}= & \left(S_{1} a_{2}+S_{2} a_{1}\right) a_{3} a_{4} e^{-\left(\lambda_{1}+\lambda_{3}\right) t}+\left(S_{3} a_{2}+S_{4} a_{1}\right) a_{4}^{2} e^{-\left(\lambda_{1}+\lambda_{4}\right) t}+\left(S_{5} a_{2}+S_{6} a_{1}\right) a_{3} a_{4} e^{-\left(\lambda_{2}+\lambda_{3}\right) t} \\
& +\left(S_{7} a_{2}+S_{8} a_{1}\right) a_{4}^{2} e^{-\left(\lambda_{2}+\lambda_{4}\right) t}+S_{9} a_{3} a_{4}^{2} e^{-\left(\lambda_{3}+\lambda_{4}\right) t}+S_{10} a_{4}^{3} e^{-2 \lambda_{4} t},
\end{aligned}
$$


where

$$
\begin{aligned}
& r_{1}=-n_{1} \lambda_{2} \lambda_{3}\left(\lambda_{1}+\lambda_{2}+2 \lambda_{3}\right) \text {, } \\
& r_{2}=-\frac{1}{2} n_{2} \lambda_{2}\left(2 \lambda_{4}^{2}+2 \lambda_{3} \lambda_{4}+\lambda_{1} \lambda_{3}+\lambda_{2} \lambda_{3}+\lambda_{1} \lambda_{4}+\lambda_{2} \lambda_{4}\right), \\
& r_{3}=-n_{3} \lambda_{2} \lambda_{4}\left(\lambda_{1}+\lambda_{2}-2 \lambda_{3}+4 \lambda_{4}\right), \\
& i_{1}=-\frac{r_{1}}{2 \lambda_{3}\left(\lambda_{1}+\lambda_{3}\right)\left(\lambda_{1}+2 \lambda_{3}-\lambda_{4}\right)}, \\
& i_{2}=-\frac{r_{1}}{2 \lambda_{3}\left(\lambda_{1}+\lambda_{3}\right)\left(\lambda_{1}+2 \lambda_{3}-\lambda_{4}\right)}\left(\frac{1}{2 \lambda_{3}}+\frac{1}{\left(\lambda_{1}+\lambda_{3}\right)}+\frac{1}{\left(\lambda_{1}+2 \lambda_{3}-\lambda_{4}\right)}\right) \text {, } \\
& i_{3}=-\frac{r_{2}}{\left(\lambda_{1}+\lambda_{3}\right)\left(\lambda_{1}+\lambda_{4}\right)\left(\lambda_{3}+\lambda_{4}\right)}, \\
& i_{4}=-\frac{r_{2}}{\left(\lambda_{1}+\lambda_{3}\right)\left(\lambda_{1}+\lambda_{4}\right)\left(\lambda_{3}+\lambda_{4}\right)}\left(\frac{1}{\left(\lambda_{1}+\lambda_{3}\right)}+\frac{1}{\left(\lambda_{1}+\lambda_{4}\right)}+\frac{1}{\left(\lambda_{3}+\lambda_{4}\right)}\right) \text {, } \\
& i_{5}=-\frac{r_{3}}{2 \lambda_{4}\left(\lambda_{1}+\lambda_{4}\right)\left(\lambda_{1}+2 \lambda_{4}-\lambda_{3}\right)} \text {, } \\
& i_{6}=-\frac{r_{3}}{2 \lambda_{4}\left(\lambda_{1}+\lambda_{4}\right)\left(\lambda_{1}+2 \lambda_{4}-\lambda_{3}\right)}\left(\frac{1}{2 \lambda_{4}}+\frac{1}{\left(\lambda_{1}+\lambda_{4}\right)}+\frac{1}{\left(\lambda_{1}+2 \lambda_{4}-\lambda_{3}\right)}\right) \text {, } \\
& m_{1}=n_{1}\left\{\left(\lambda_{1}+2 \lambda_{3}\right)\left(\lambda_{1}+2 \lambda_{3}-\lambda_{4}\right)+\lambda_{3}\left(\lambda_{1}+\lambda_{2}+2 \lambda_{3}\right)\right\}, \quad m_{2}=-\frac{3}{2}, \\
& m_{3}=n_{1}\left(2 \lambda_{3}+\lambda_{2}\right)\left(2 \lambda_{3}+\lambda_{2}-\lambda_{4}\right), \quad m_{4}=-\frac{3}{2}, \\
& s_{1}=\frac{1}{2} n_{2}\left\{\left(\lambda_{1}+\lambda_{3}\right)\left(2 \lambda_{1}+\lambda_{3}+3 \lambda_{4}\right)+\left(2 \lambda_{4}^{2}+2 \lambda_{3} \lambda_{4}+\lambda_{1} \lambda_{3}+\lambda_{2} \lambda_{3}+\lambda_{1} \lambda_{4}+\lambda_{2} \lambda_{4}\right)\right\}, \\
& s_{2}=-3, s_{3}=n_{3}\left\{\left(\lambda_{1}+\lambda_{4}\right)\left(\lambda_{1}-\lambda_{3}+3 \lambda_{4}\right)+\lambda_{4}\left(\lambda_{1}+\lambda_{2}-2 \lambda_{3}+4 \lambda_{4}\right)\right\} \text {, } \\
& s_{4}=-\frac{3}{2}, s_{5}=\frac{1}{2} n_{2}\left(\lambda_{2}+\lambda_{3}\right)\left(2 \lambda_{2}+\lambda_{3}+3 \lambda_{4}\right), \quad s_{6}=-3 \text {, } \\
& s_{7}=n_{3}\left(\lambda_{2}+\lambda_{4}\right)\left(\lambda_{2}-\lambda_{3}+3 \lambda_{4}\right), \quad s_{8}=-\frac{3}{2}, \\
& M_{1}=-\frac{m_{1}}{2 \lambda_{3}\left(\lambda_{1}+2 \lambda_{3}-\lambda_{2}\right)\left(\lambda_{1}+2 \lambda_{3}-\lambda_{4}\right)}, \quad M_{2}=-\frac{m_{2}}{2 \lambda_{3}\left(\lambda_{1}+2 \lambda_{3}-\lambda_{2}\right)\left(\lambda_{1}+2 \lambda_{3}-\lambda_{4}\right)}, \\
& M_{3}=-\frac{m_{3}}{2 \lambda_{3}\left(\lambda_{2}+2 \lambda_{3}-\lambda_{1}\right)\left(\lambda_{2}+2 \lambda_{3}-\lambda_{4}\right)}, \quad M_{4}=-\frac{m_{4}}{2 \lambda_{3}\left(\lambda_{2}+2 \lambda_{3}-\lambda_{1}\right)\left(\lambda_{2}+2 \lambda_{3}-\lambda_{4}\right)}, \\
& M_{5}=\frac{1}{\left(\lambda_{1}-3 \lambda_{3}\right)\left(\lambda_{2}-3 \lambda_{3}\right)\left(3 \lambda_{3}-\lambda_{4}\right)}, \quad M_{6}=\frac{3}{2 \lambda_{3}\left(\lambda_{1}-2 \lambda_{3}-\lambda_{4}\right)\left(\lambda_{2}-2 \lambda_{3}-\lambda_{4}\right)} \text {, } \\
& S_{1}=-\frac{s_{1}}{\left(\lambda_{1}+\lambda_{4}\right)\left(\lambda_{3}+\lambda_{4}\right)\left(-\lambda_{1}+\lambda_{2}-\lambda_{3}-\lambda_{4}\right)}, \quad S_{2}=-\frac{s_{2}}{\left(\lambda_{1}+\lambda_{4}\right)\left(\lambda_{3}+\lambda_{4}\right)\left(-\lambda_{1}+\lambda_{2}-\lambda_{3}-\lambda_{4}\right)}, \\
& S_{3}=\frac{s_{3}}{2 \lambda_{4}\left(\lambda_{1}-\lambda_{2}+2 \lambda_{4}\right)\left(-\lambda_{1}+\lambda_{3}-2 \lambda_{4}\right)}, \quad S_{4}=\frac{s_{4}}{2 \lambda_{4}\left(\lambda_{1}-\lambda_{2}+2 \lambda_{4}\right)\left(-\lambda_{1}+\lambda_{3}-2 \lambda_{4}\right)}, \\
& S_{5}=-\frac{s_{5}}{\left(\lambda_{2}+\lambda_{4}\right)\left(\lambda_{3}+\lambda_{4}\right)\left(\lambda_{1}-\lambda_{2}-\lambda_{3}-\lambda_{4}\right)}, \quad S_{6}=-\frac{s_{6}}{\left(\lambda_{2}+\lambda_{4}\right)\left(\lambda_{3}+\lambda_{4}\right)\left(\lambda_{1}-\lambda_{2}-\lambda_{3}-\lambda_{4}\right)},
\end{aligned}
$$




$$
\begin{array}{ll}
S_{7}=-\frac{s_{7}}{2 \lambda_{4}\left(\lambda_{1}-\lambda_{2}-2 \lambda_{4}\right)\left(\lambda_{2}-\lambda_{3}+2 \lambda_{4}\right)}, & S_{8}=-\frac{s_{8}}{2 \lambda_{4}\left(\lambda_{1}-\lambda_{2}-2 \lambda_{4}\right)\left(\lambda_{2}-\lambda_{3}+2 \lambda_{4}\right)}, \\
S_{9}=\frac{3}{2 \lambda_{4}\left(\lambda_{1}-\lambda_{3}-2 \lambda_{4}\right)\left(\lambda_{2}-\lambda_{3}-2 \lambda_{4}\right)}, & S_{10}=-\frac{1}{\left(\lambda_{1}-3 \lambda_{4}\right)\left(\lambda_{2}-3 \lambda_{4}\right)\left(\lambda_{3}-3 \lambda_{4}\right)} .
\end{array}
$$

Here $u_{1}$ is a correction term and has also very small contribution in the solution. However it is laborious work to solve the Eq. (9) for $u_{1}$. So we neglect the calculation of $u_{1}$. Putting the values of $A_{1}, A_{2}, A_{3}$ and $A_{4}$ from Eqs. (21), (16), (22), (23) into Eq. (4) and integrating, we obtain

$$
\begin{aligned}
& a_{1}(t)=a_{1,0}+\varepsilon\left[a_{2,0} a_{3,0}^{2} \times\left\{\begin{array}{l}
i_{2}\left(1-e^{\left(-\lambda_{1}+\lambda_{2}-2 \lambda_{3}\right) t}\right) \\
\left.-i_{1}\left(t e^{\left(-\lambda_{1}+\lambda_{2}-2 \lambda_{3}\right) t}+\frac{e^{\left(-\lambda_{1}+\lambda_{2}-2 \lambda_{3}\right) t}-1}{\lambda_{1}-\lambda_{2}+2 \lambda_{3}}\right)\right\} /\left(\lambda_{1}-\lambda_{2}+2 \lambda_{3}\right)
\end{array}\right.\right. \\
& +a_{2,0} a_{3,0} a_{4,0} \times\left\{\begin{array}{l}
i_{4}\left(1-e^{\left(-\lambda_{1}+\lambda_{2}-\lambda_{3}-\lambda_{4}\right) t}\right) \\
-i_{3}\left(t e^{\left(-\lambda_{1}+\lambda_{2}-\lambda_{3}-\lambda_{4}\right) t}+\frac{e^{\left(-\lambda_{1}+\lambda_{2}-\lambda_{3}-\lambda_{4}\right) t}-1}{\lambda_{1}-\lambda_{2}+\lambda_{3}+\lambda_{4}}\right)
\end{array}\right\} /\left(\lambda_{1}-\lambda_{2}+\lambda_{3}+\lambda_{4}\right) \\
& +a_{2,0} a_{4,0}^{2} \times\left\{\begin{array}{l}
i_{6}\left(1-e^{\left(-\lambda_{1}+\lambda_{2}-2 \lambda_{4}\right) t}\right) \\
\left.-i_{5}\left(t e^{\left(-\lambda_{1}+\lambda_{2}-2 \lambda_{4}\right) t}+\frac{e^{\left(-\lambda_{1}+\lambda_{2}-2 \lambda_{4}\right) t}-1}{\lambda_{1}-\lambda_{2}+2 \lambda_{4}}\right)\right\} /\left(\lambda_{1}-\lambda_{2}+2 \lambda_{4}\right)
\end{array}\right], \\
& a_{2}(t)=a_{2,0}+\varepsilon a_{2,0}\left[n_{1} a_{3,0}^{2}\left(\frac{1-e^{-2 \lambda_{3} t}}{2 \lambda_{3}}\right)+n_{2} a_{3,0} a_{4,0}\left(\frac{1-e^{-\left(\lambda_{3}+\lambda_{4}\right) t}}{\lambda_{3}+\lambda_{4}}\right)+n_{3} a_{4,0}^{2}\left(\frac{1-e^{-2 \lambda_{4} t}}{2 \lambda_{4}}\right)\right] \text {, } \\
& a_{3}(t)=a_{3,0}+\varepsilon\left[a_{3,0}^{2}\left\{M_{1} a_{2,0}+M_{2} a_{1,0}\right\}\left(\frac{1-e^{-\left(\lambda_{1}+\lambda_{3}\right) t}}{\lambda_{1}+\lambda_{3}}\right)+a_{3,0}^{2}\left\{M_{3} a_{2,0}+M_{4} a_{1,0}\right\}\left(\frac{1-e^{-\left(\lambda_{2}+\lambda_{3}\right) t}}{\lambda_{2}+\lambda_{3}}\right)\right. \\
& \left.+a_{3,0}^{3} M_{5}\left(\frac{1-e^{-2 \lambda_{3} t}}{2 \lambda_{3}}\right)+a_{3,0}^{2} a_{4} M_{6}\left(\frac{1-e^{-\left(\lambda_{3}+\lambda_{4}\right) t}}{\lambda_{3}+\lambda_{4}}\right)\right] \\
& a_{4}(t)=a_{4,0}+\varepsilon\left[a_{3,0} a_{4,0}\left\{S_{1} a_{2,0}+S_{2} a_{1,0}\right\}\left(\frac{1-e^{-\left(\lambda_{1}+\lambda_{3}\right) t}}{\lambda_{1}+\lambda_{3}}\right)+a_{4,0}^{2}\left\{S_{3} a_{2,0}+S_{4} a_{1,0}\right\}\left(\frac{1-e^{-\left(\lambda_{1}+\lambda_{4}\right) t}}{\lambda_{1}+\lambda_{4}}\right)\right. \\
& +a_{3,0} a_{4,0}\left\{S_{5} a_{2,0}+S_{6} a_{1,0}\right\}\left(\frac{1-e^{-\left(\lambda_{2}+\lambda_{3} t\right.}}{\lambda_{2}+\lambda_{3}}\right)+a_{4,0}^{2}\left\{S_{7} a_{2,0}+S_{8} a_{1,0}\right\}\left(\frac{1-e^{-\left(\lambda_{2}+\lambda_{4}\right) t}}{\lambda_{2}+\lambda_{4}}\right) \\
& \left.+a_{3,0} a_{4,0}^{2} S_{9}\left(\frac{1-e^{-\left(\lambda_{3}+\lambda_{4}\right) t}}{\lambda_{3}+\lambda_{4}}\right)+a_{4,0}^{3} S_{10}\left(\frac{1-e^{-2 \lambda_{4} t}}{2 \lambda_{4}}\right)\right] .
\end{aligned}
$$

Therefore, we obtain the first approximate solution of the Eq. (11) is given by

$$
x(t, \varepsilon)=\frac{1}{2} a_{1}\left(e^{-\lambda_{1} t}+e^{-\lambda_{2} t}\right)+a_{2}\left(\frac{e^{-\lambda_{1} t}-e^{-\lambda_{2} t}}{\lambda_{1}-\lambda_{2}}\right)+a_{3} e^{-\lambda_{3} t}+a_{4} e^{-\lambda_{4} t}
$$

where $a_{1}, a_{2}, a_{3}, a_{4}$ are given by the Eq. (25). 


\section{RESULTS AND DISCUSSION}

To test the accuracy of the approximate analytical solutions obtained by the presented technique have been compared to the numerical solutions. Firstly, $x(t, \varepsilon)$ is calculated by the Eq. (26) by using the imposed conditions $\lambda_{1} \rightarrow \lambda_{2}, \lambda_{4} \approx \lambda_{3}+2 \lambda_{1}$ but $\lambda_{4}\left\langle 2 \lambda_{3}\right.$ in which $a_{1}, a_{2}, a_{3}, a_{4}$ are calculated by the Eq. (25). Moreover, if we replace $-\varepsilon f(x)$ by $\varepsilon f(x)$ with $\varepsilon<<1$, then the analytical approximate solutions will also be conversed to the corresponding numerical solutions. The corresponding numerical solutions of Eq. (11) are computed by fourth order Runge-Kutta method. The analytical approximate solutions and numerical solutions are plotted in Fig. 1 and Fig. 2 for different initial conditions.

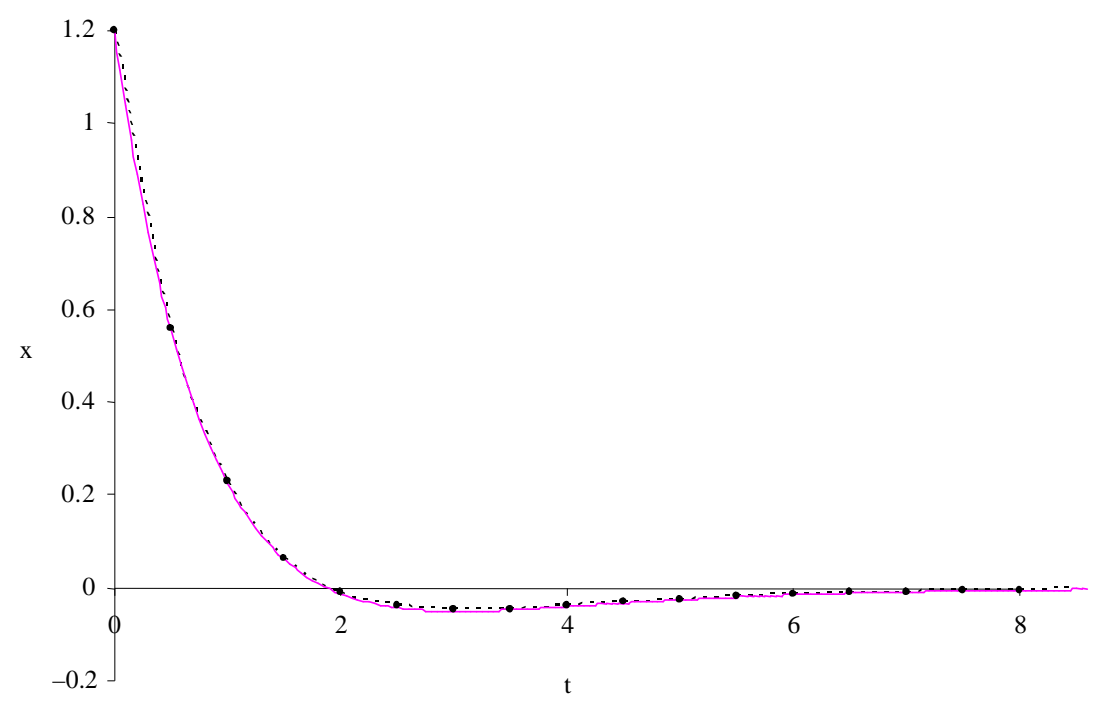

Fig.1. First approximate solution of Eq. (11) is denoted by $-\bullet-$ (dashed lines) by the presented method with the initial conditions $a_{1,0}=0.6, a_{2,0}=0.6, a_{3,0}=0.6, a_{4,0}=0.6$ or $[x(0)=1.80000, \dot{x}(0)=-2.61561, \ddot{x}(0)=3.33514, \dddot{x}(0)=-3.88353]$ when $\lambda_{1}=0.7$, $\lambda_{2}=0.95, \lambda_{3}=1.18, \lambda_{4}=1.35, \varepsilon=0.1$ and $f=x^{3}$. Corresponding numerical solution is denoted by - (solid line). 


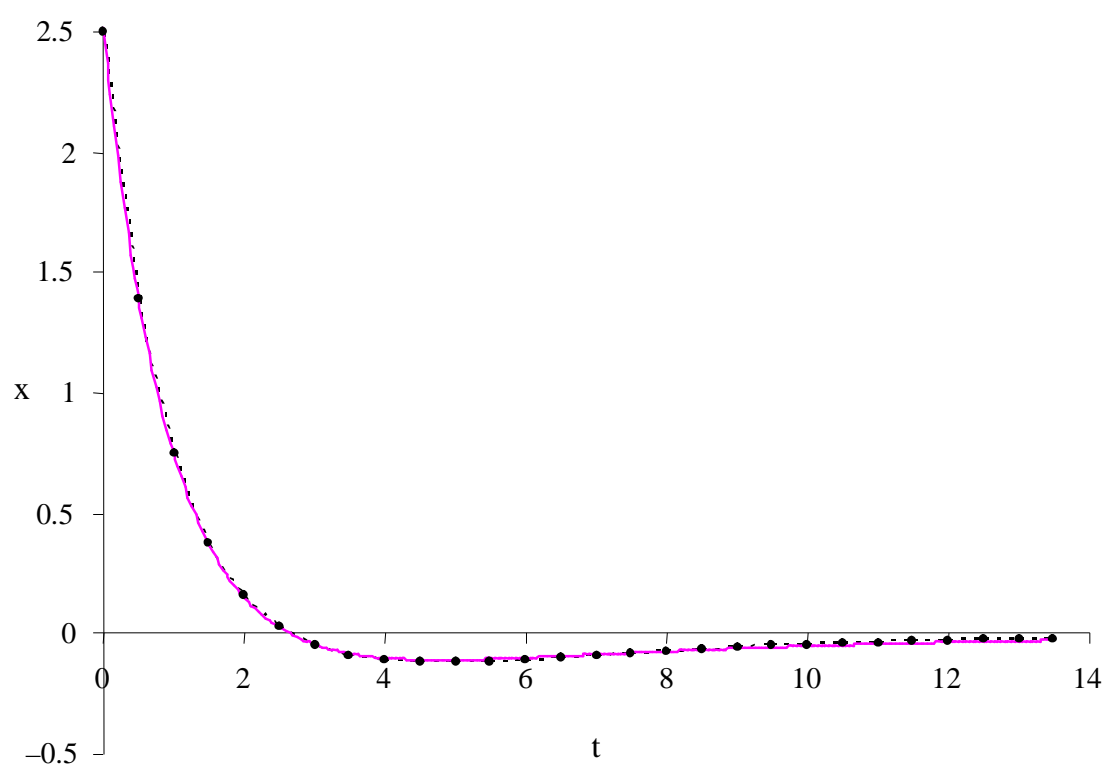

Fig. 2. First approximate solution of Eq. (11) is denoted by - - - (dashed lines) by the presented method with the initial conditions $a_{1,0}=1.0, a_{2,0}=0.5, a_{3,0}=1.0, a_{4,0}=0.5$ or $[x(0)=2.50000, \dot{x}(0)=-2.88042, \ddot{x}(0)=3.13488, \dddot{x}(0)=-3.07011]$ when $\lambda_{1}=0.25, \lambda_{2}=0.8$, $\lambda_{3}=1.2, \lambda_{4}=1.43, \varepsilon=0.1$ and $f=x^{3}$. Corresponding numerical solution is denoted by (solid line).

\section{CONCLUSION}

The KBM method has been extended for solving the fourth order near critically damped nonlinear systems under some special conditions with small nonlinearities, when the four eigenvalues of the corresponding linear equation are real and negative numbers. It is also noted that the analytical approximate solutions will be conversed to the corresponding numerical solutions obtained by the fourth order Rangue-Kutta method whether the small parameter is positive or negative. From the Figs $1-2$, it is noticed that the solutions obtained by the presented method show good agreement with those obtained by the numerical method.

\section{REFERENCES}

Akbar, M. A., M. S. Uddin, M. R. Islam and A. A. Soma. 2007. Krylov-Bogoliubov-Mitropolskii (KBM) Method for Fourth Order More Critically Damped Nonlinear Systems. J. Mech. of Continua and Math. Sciences 2(1): 91-107.

Alam, M. S. and M. A. Sattar. 1996. An Asymptotic Method for Third Order Critically Damped Nonlinear Equations. J. Mathematical and Physical Sciences 30: 291-298. 
Alam, M. S. 2002. Asymptotic Method for Non-oscillatory Nonlinear Systems. Far East J. Appl. Math. 7: 119-128.

Bogoliubov, N. N. and Yu. A. Mitropolskii. 1961. Asymptotic Methods in the Theory of Nonlinear Oscillations. Gordan and Breach, New York.

Haque, B. M. I., M. A. Alom, and M. H. Rahman. 2011. Perturbation Solution of Fourth Order Critically Damped Oscillatory Nonlinear Systems. International Journal of Basic \& Applied Sciences 11(3): 68-77.

Krylov, N. N. and N. N. Bogoliubov. 1947. Introduction to Nonlinear Mechanics. Princeton University Press, New Jersey.

Murty, I. S. N., B. L. Deekshatulu and G. Krishna. 1969. On an Asymptotic Method of KrylovBogoliubov for Over-damped Nonlinear Systems, J. Frank. Inst. 288: 49-65.

Murty, I. S. N. 1971. A Unified Krylov-Bogoliubov method for Solving Second Order Nonlinear Systems. Int. J. Nonlinear Mech. 6: 45-53.

Popov, I. P. 1956. A Generalization of the Bogoliubov Asymptotic Method in the Theory of Nonlinear Oscillations (in Russian), Dokl. Akad. USSR 3: 308-310.

Rahman, M. H., B. M. I. Haque and M. A. Akbar. 2009. Asymptotic Solutions of Fourth Order Near Critically Damped Nonlinear Systems. Journal of Informatics and Mathematical Sciences 1(1): 61-73.

(Received revised manuscript on 8 May, 2012) 